\title{
Rolling tachyon boundary conformal field theory on an orbifold
}

\section{AUTHOR(S):}

Kawai, S; Keski-Vakkuri, E; Leigh, RG; Nowling, S

\section{CITATION:}

Kawai, S ... [et al]. Rolling tachyon boundary conformal field theory on an orbifold. PHYSICAL REVIEW D 2006, 73(10): 106003.

\section{ISSUE DATE:}

2006-05

URL:

http://hdl.handle.net/2433/50448

RIGHT:

Copyright 2006 American Physical Society 
PHYSICAL REVIEW D 73, 106003 (2006)

\title{
Rolling tachyon boundary conformal field theory on an orbifold
}

\author{
Shinsuke Kawai, ${ }^{1, *}$ Esko Keski-Vakkuri, ${ }^{2, \dagger}$ Robert G. Leigh, ${ }^{3, \ddagger}$ and Sean Nowling ${ }^{3, \S}$ \\ ${ }^{1}$ YITP, Kyoto University, Kyoto 606-8502, Japan \\ ${ }^{2}$ Helsinki Institute of Physics and Department of Physical Sciences, P.O. Box 64, FIN-00014 University of Helsinki, Finland \\ ${ }^{3}$ Department of Physics, University of Illinois at Urbana-Champaign, 1110 West Green Street, Urbana, Illinois 61801-3080, USA
}

(Received 22 February 2006; published 8 May 2006)

\begin{abstract}
We consider the nontrivial boundary conformal field theory with exactly marginal boundary deformation. In recent years this deformation has been studied in the context of rolling tachyons and $S$-branes in string theory. Here we study the problem directly from an open string point of view, at one loop. We formulate the theory of the $\mathbb{Z}_{2}$ reflection orbifold. To do so, we extend fermionization techniques originally introduced by Polchinski and Thorlacius. We also explain how to perform the open string computations at arbitrary (rational) radius, by consistently constructing the corresponding shift orbifold, and show in what sense these are related to known boundary states. In a companion paper, we use these results in a cosmological context involving decaying branes.
\end{abstract}

PACS numbers: $11.25 . \mathrm{Hf}$

\section{INTRODUCTION}

The $c=1$ conformal field theory on world sheets with boundary is known to have a boundary interaction

$$
-\frac{\lambda}{2} \int_{\partial \Sigma} d s e^{i X / \sqrt{\alpha^{\prime}}}+\text { h.c. }
$$

which is exactly marginal. This theory was originally studied at self-dual radius $\left(X \in S_{R=\sqrt{\alpha^{\prime}}}^{1}\right.$ ) by Callan et al. [1], where the marginality was established. Although there is renormalization of $\lambda$ in perturbation theory, due to collisions of $e^{i X / \sqrt{\alpha^{\prime}}}$ with $e^{-i X / \sqrt{\alpha^{\prime}}}$, it is possible to absorb these divergences and obtain an RG-stationary coupling. At self-dual radius, the operators of the theory organize themselves into multiplets of an $\widehat{S U(2)})_{1}$ current algebra, and this structure plays an important organizing rôle in the analysis.

A complementary analysis of this theory, at infinite radius, was later provided by Polchinski and Thorlacius [2]. By introducing auxiliary bosonic fields, it is possible to rewrite the theory in terms of free fermions with a quadratic boundary interaction. This essentially constitutes a regularization of the theory (different than the one noted above) and is tractable because the action is quadratic in fermions (from the bulk world sheet point of view, there are mass terms with $\delta$-function support-these are both classically and quantum mechanically marginal).

In this paper, we will consider the extension of this theory to other backgrounds, including a $\mathbb{Z}_{2}$ reflection orbifold, as well as circles of arbitrary rational radius. We were led into this work by considerations of $S$-brane solutions [3-5] in Lorentzian orbifold backgrounds [6,7]. In a companion paper [8] (see also [9]), we use the results

\footnotetext{
*Electronic address: skawai@yukawa.kyoto-u.ac.jp

${ }^{\dagger}$ Electronic address: esko.keski-vakkuri@helsinki.fi

Electronic address: rgleigh@uiuc.edu

${ }^{\S}$ Electronic address: nowling@students.uiuc.edu
}

of the present paper; following a Wick rotation, the results are applicable to the discussion of "fractional $S$-branes," objects which may be of importance in cosmological orbifold backgrounds.

Our calculations will be formulated and presented directly in the open string channel. Passing these results to the closed string channel then allows for the determination of boundary states. ${ }^{1}$ Away from self-dual radius, we must formulate the theories of interest in the fermionic picture. In particular, this was originally formulated at infinite radius. For the orbifold theory, it is necessary to carefully consider various subtleties of the fermionic construction. As a result, we have organized the paper as follows. In Sec. II, we set up notation and discuss some standard boundary states of the undeformed theory. Then, in Sec. III, we review the standard bosonic treatment of the bosonic theory at self-dual radius. In Sec. IV we then review the fermionic construction of the infinite radius deformed theory. As we have mentioned above, there are a number of subtleties involved in extending this analysis to the orbifold theory, and thus we take the liberty of going into some detail in this review. In this section we also review how the corresponding boundary states can be written in terms of a projection operator acting on $\widehat{S U(2)})_{1}$ Ishibashi states. We then discuss how finite radius theories may be constructed in the fermionic picture. In constructing these, there are both classical and quantum consistency conditions in the fermionic path integral to which we must pay attention. Doing so gives rise in the end to boundary states that can be written using projection operators, and these boundary states transform in a simple way under $T$-duality. In Sec. V, we construct the orbifold theory in the fermionic description. In so doing, we intro-

\footnotetext{
${ }^{1}$ For related work on boundary states on deformed boundary conformal field theory on the orbifold, see also [10]. Stable nonBPS branes on a $T^{4}$ orbifold have been considered in [11].
} 
duce a number of consistency checks to ensure that the results are correct.

The boundary states, including contributions from both twisted and untwisted sectors, may be constructed at arbitrary radius. In the companion paper [8], we construct all of the fractional boundary states, and show that they have a consistent interpretation. In particular, we can see explicitly that although the finite radius theory has two orbifold fixed points, the infinite radius theory has but one. This is reflected nicely in the structure of the boundary states.

\section{BOUNDARY CFT OF A FREE BOSON}

In order to set notation and collect some known results, we first consider the undeformed boundary conformal theory on a circle of radius $R$. Free open bosonic string theory, with action $\frac{1}{4 \pi \alpha^{\prime}} \int d^{2} \sigma \sqrt{-\bar{h}} h^{a b} \partial_{a} X \partial_{b} X$, on a strip $\sigma \in(0, r), \tau \in(0, \ell)$, has mode expansion

$$
\begin{aligned}
\hat{X}(\sigma, \tau)= & \hat{x}+\frac{2 \pi \alpha^{\prime}}{r} \tau \hat{p} \\
& +i \sqrt{2 \alpha^{\prime}} \sum_{\substack{n \in \mathbb{Z} \\
n \neq 0}} \frac{1}{n} \hat{\alpha}_{n} \cos \left(\frac{\pi n \sigma}{r}\right) e^{-i \pi n \tau / r}
\end{aligned}
$$

where we have assumed Neumann boundary conditions at $\sigma=0, r$. The spectrum of $\hat{p}$ is determined by the compactification radius, spectrum $(\hat{p})=\mathbb{Z} / R$. With this normalization, the vertex operator $e^{i k X}$ has conformal dimension $\Delta=\alpha^{\prime} k^{2}$.

If we compute the $\mathrm{NN}$ annulus amplitude (the open string partition function with Neumann boundary conditions on each boundary), we may obtain information on the Neumann boundary state of the closed string channel. This is

$$
\mathcal{A}_{\mathrm{NN}}=\frac{1}{\eta(q)} \sum_{n} q^{\alpha^{\prime} n^{2} / R^{2}}
$$

We use the notation $q=e^{-\pi t}, t=\ell / r$. This may be rewritten as

$$
\mathcal{A}_{\mathrm{NN}}=\frac{R}{\eta(q)} \int d p q^{\alpha^{\prime} p^{2}} \sum_{m} e^{2 \pi i p R m},
$$

which can be understood as explicitly implementing the shift orbifold to finite radius within the infinite radius theory. This form will be important later.

In the present $\mathrm{NN}$ case, at finite radius, it is also possible to introduce a Wilson line, and we record the result [12] here

$$
\mathcal{A}_{\mathrm{NN}}(\Delta \theta)=\frac{1}{\eta(q)} \sum_{n} q^{\alpha^{\prime}(n / R+\Delta \theta / 2 \pi R)^{2}} .
$$

By Poisson resummation, with notation $\tilde{q}=e^{-2 \pi / t}$, we find

$$
\mathcal{A}_{\mathrm{NN}}(\Delta \theta)=\frac{R}{\sqrt{2 \alpha^{\prime}} \eta\left(\tilde{q}^{2}\right)} \sum_{m \in \mathbb{Z}}\left(\tilde{q}^{2}\right)^{m^{2} R^{2} / 4 \alpha^{\prime}} e^{-i m \Delta \theta} .
$$

In this channel, we identify boundary states via [13]

$$
\mathcal{A}_{\mathrm{NN}}(\Delta \theta) \equiv\langle N, \theta|\Delta(\tilde{q})| N, \theta+\Delta \theta\rangle
$$

with $\Delta(\tilde{q})$ the closed string propagator. We may write the boundary state in oscillator form as [12]

$$
|N, \theta\rangle=2^{-1 / 4} e^{\sum_{k} \alpha_{k} \tilde{\alpha}_{k}}|0\rangle_{\text {Fock }} \otimes \sum_{n \in \mathbb{Z}} e^{i n \theta}\left|\frac{n R}{\sqrt{\alpha^{\prime}}},-\frac{n R}{\sqrt{\alpha^{\prime}}}\right\rangle
$$

In this form, it is clear that the Neumann boundary state has zero momentum, ${ }^{2}$ and is at fixed $\tilde{X} \equiv X_{L}-X_{R}$.

Note that at self-dual radius, $R=\sqrt{\alpha^{\prime}}$, the conformal dimensions are square integers. In fact, there is an $\widehat{S U(2)}$ current algebra that classifies the spectrum (see e.g. [14]). In this case, (4) can be rewritten [15]

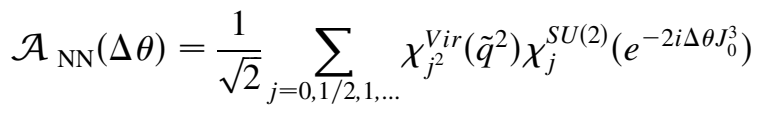

with $S U(2)$ characters

$$
\chi_{j}^{S U(2)}(g)=\operatorname{Tr}_{j} \mathcal{D}^{(j)}(g),
$$

where $\mathcal{D}^{(j)}(g)$ is the matrix representing the $S U(2)$ element $g$ in representation $j$, and Virasoro characters

$$
\chi_{j^{2}}^{\operatorname{Vir}}\left(\tilde{q}^{2}\right)=\frac{\tilde{q}^{2 j^{2}}-\tilde{q}^{2(j+1)^{2}}}{\eta\left(\tilde{q}^{2}\right)} .
$$

Using the normalization of Ishibashi states

$$
\left\langle\left\langle j, m, n|\Delta(\tilde{q})| j^{\prime}, m^{\prime}, n^{\prime}\right\rangle\right\rangle=\chi_{j^{2}}^{V i r}\left(\tilde{q}^{2}\right) \delta_{j j^{\prime}} \delta_{m m^{\prime}} \delta_{n n^{\prime}}
$$

we obtain the boundary state in the $\widehat{S U(2)}$ basis

$$
\left.|N, \theta\rangle \simeq 2^{-1 / 4} \sum_{j=0,1 / 2,1, \ldots} \sum_{m, n=-j}^{j} \mathcal{D}_{m, n}^{(j)}\left(e^{-2 i \theta J_{0}^{3}}\right)|j,-m, n\rangle\right\rangle .
$$

\section{Chan-paton factors}

With multiple branes, the above computation is only trivially modified. The boundaries attain an extra discrete index labeling the map of the world sheet boundary onto a $D$-brane. Consequently, the boundary state carries an index $k$, labeling the $D$-brane. The general construction is reviewed more fully in the companion paper [8].

\footnotetext{
${ }^{2}$ We record the Dirichlet boundary state at self-dual radius$$
|D, x\rangle \simeq 2^{-1 / 4} e^{-\sum_{k} \alpha_{k} \tilde{\alpha}_{k}}|0\rangle_{\text {Fock }} \otimes \sum_{n} e^{-i n x / \sqrt{\alpha^{\prime}}}\left|\frac{n}{\sqrt{\alpha^{\prime}}}, \frac{n}{\sqrt{\alpha^{\prime}}}\right\rangle
$$ 


\section{ADSORPTION AND OPEN STRING PARTITION FUNCTIONS}

\section{A. Boundary deformations}

In the context of rolling tachyons, the generic boundary perturbation of interest is of the form

$$
S_{\lambda}=\int_{\partial \Sigma} d s\left[\lambda_{+} e^{X^{0}(s) / \sqrt{\alpha^{\prime}}}+\lambda_{-} e^{-X^{0}(s) / \sqrt{\alpha^{\prime}}}\right]
$$

where $X^{0}$ is the timelike target space coordinate. Classically (using the correlators of the undeformed theory), this perturbation is marginal, that is $d_{\mathrm{cl}}=1$. For $\lambda_{ \pm}=\frac{1}{2} \lambda e^{\mp X_{0}^{0} / \sqrt{\alpha^{\prime}}}$, this is related to the "full $S$-brane" [3] centered at $X_{0}^{0}$, while for $\lambda_{-}=0$, we have the "half $S$-brane" [5]. The full $S$-brane corresponds to a process where a carefully fine-tuned initial closed string configuration time evolves to form an unstable $D$-brane which then decays to a final state of closed strings [16-19]. The whole process is centered around the time $X_{0}^{0}$ and is time reflection invariant about it, as evident from writing the deformation in the form

$$
S_{\lambda}=\lambda \int_{\partial \Sigma} d s \cosh \left[\left(X^{0}(s)-X_{0}^{0}\right) / \sqrt{\alpha^{\prime}}\right]
$$

in particular the initial state of closed strings is a time reflection image of the final state. By Wick rotating $X^{0}=$ $i X$, it becomes

$$
S_{\lambda}=-\lambda \int_{\partial \Sigma} d s \cos \left[\left(X(s)-X_{0}\right) / \sqrt{\alpha^{\prime}}\right],
$$

which is a known exactly marginal deformation [1]. In practice, computations in the background (12) are first performed in the Euclidean signature with (13), and the results are then Wick rotated back to the Lorentzian signature. In the rest of the present paper, we will disregard any relation to rolling tachyons, and simply consider the Euclidean theory.

One could absorb the parameter $X_{0}$ into the definition of the origin of time. However, for a given world sheet with multiple boundaries, there can be a distinct deformation for each boundary component. For example, if we consider the annulus, we will consider a boundary deformation of the form

$$
\begin{aligned}
S_{\mathrm{int}}= & -\lambda \int_{\partial \Sigma_{1}} d s \cos \left(\frac{X-X_{0}^{(1)}}{\sqrt{\alpha^{\prime}}}\right) \\
& -\tilde{\lambda} \int_{\partial \Sigma_{2}} d s \cos \left(\frac{X-X_{0}^{(2)}}{\sqrt{\alpha^{\prime}}}\right)
\end{aligned}
$$

where $\partial \Sigma_{j}$ are the boundary components. This is essentially a Chan-Paton structure. Indeed in the presence of multiple branes, $\lambda$ and $\tilde{\lambda}$ would be replaced by matrices, and the annulus would include overall traces for each boundary component. A priori, there is no need to take the cosines to be centered at the same point on different boundaries, and the difference cannot be absorbed to the choice of the time origin.

In the orbifold $\mathbb{R}^{1, d} / \mathbb{Z}_{2}$ the $\mathbb{Z}_{2}$ acts by $\left(X^{0}, X^{1}, \ldots, X^{d}\right) \rightarrow-\left(X^{0}, X^{1}, \ldots, X^{d}\right)$ [6,7]. After Wick rotation $X^{0}=i X$, we obtain a Euclidean orbifold $\mathbb{R}^{d+1} / \mathbb{Z}_{2}, \quad$ where $\mathbb{Z}_{2}$ acts by $\left(X, X^{1}, \ldots, X^{d}\right) \rightarrow$ $-\left(X, X^{1}, \ldots, X^{d}\right)$. The full $S$-brane deformation is invariant under the orbifold identifications, if we choose it to be centered around $X^{0}=0$. In the Euclidean signature, for world sheets with multiple boundaries, if we allow for distinct deformations at each boundary component $\partial \Sigma_{j}$, we would then need each of them to be centered around $X=0$ (i.e., set $X_{0}^{(j)}=0$, but the associated parameters $\lambda_{j}$ can be independent of one another. Wick rotation back to Lorentzian signature is subtle, because of the issues with the branching of time's arrow. This will be discussed in Ref. [8].

\section{B. The adsorption method}

Let us begin with the theory at self-dual radius $R_{\mathrm{sd}}=$ $\sqrt{\alpha^{\prime}}$. One way to understand this case is known as "adsorption" [20]. This method highlights the close relationship with the Kondo problem of condensed matter physics. In that situation we think of the deformation as the $U(1)$ charge current for a free fermionic theory.

We can study this theory by replacing the boson $\hat{X}$ on the strip by an "unfolded" chiral boson $\hat{\phi}$ on $\sigma \in(-r, r)$, $\tau \in(0, \ell)$, where $\sigma=-r$ is identified with $\sigma=+r$ (this is a torus in the $w=i \tau+\sigma$ plane, with modular parameter $\ell / 2 r)$. To do so, we identify $\phi(\sigma, \tau)=X_{L}(i \tau+\sigma)$ for $\sigma>0$, and $\phi(\sigma, \tau)=X_{R}(i \tau-\sigma)$ for $\sigma<0$. It is most natural to quantize this boson by taking $\sigma$ as the "time" direction; we simply have a periodic boundary condition in the $\tau$-direction, and we obtain the mode expansion

$$
\hat{\phi}(w)=\hat{\phi}_{0}+\frac{\pi \alpha^{\prime} w \hat{p}}{\ell}+i \sqrt{\frac{\alpha^{\prime}}{2}} \sum_{\substack{n \in \mathbb{Z} \\ n \neq 0}} \frac{1}{n} \hat{\alpha}_{n} e^{-2 \pi n w / \ell} .
$$

At self-dual radius, the theory reduces to a chiral $\widehat{S U(2)}$ current algebra, with currents

$$
J^{ \pm}=e^{ \pm i \phi / \sqrt{\alpha^{\prime}}} \quad J^{3}=i \partial \phi / \sqrt{\alpha^{\prime}},
$$

where $J^{ \pm}=\left(J^{1} \pm J^{2}\right) / \sqrt{2}$. The basic strategy will be to make use of the $\widehat{S U(2)}$ current algebra relations,

$$
\mathcal{H}_{0} \propto:\left(J^{3}(\sigma)\right)^{2}:=\frac{1}{3}: \vec{J}^{2}(\sigma):=:\left(J^{1}(\sigma)\right)^{2}: .
$$

For simplicity we will focus on the situation with a single boundary deformation, taking the form

$$
\lambda \int_{0}^{\ell} d \tau J^{1}(i \tau+0)=\ell \lambda J_{0}^{1} .
$$

In detail, the partition function becomes: 


$$
\begin{aligned}
Z_{\lambda}= & \operatorname{Tr}\left(e^{1 / 2 \pi \int_{-r}^{0} d \sigma \int_{0}^{\ell} d \tau:\left(J^{1}(\sigma, \tau)\right)^{2}:} e^{i \lambda \int_{0}^{\ell} d \tau J^{1}(\tau+i 0)}\right. \\
& \left.\times e^{1 / 2 \pi \int_{0}^{r} d \sigma \int_{0}^{\ell} d \tau:\left(J^{1}(\sigma, \tau)\right)^{2}:}\right) .
\end{aligned}
$$

Here the boundary deformation takes the form of an operator insertion at the fixed "time" $\sigma=0$. Using the explicit mode expansions, we obtain

$$
\begin{aligned}
Z_{\lambda} & =\operatorname{Tr}\left(e^{-(r / \ell \pi) \cdot\left(\left(J_{0}^{1}\right)^{2}+2 \sum_{n=1}^{\infty} J_{-n}^{1} J_{n}^{1}\right)} e^{\left.i \lambda J_{0}^{1}\right)}\right. \\
& =\frac{1}{\eta\left(\tilde{q}^{2}\right)} \sum_{n \in \mathbb{Z}}\left(\tilde{q}^{2}\right)^{n^{2} / 4} e^{\pi i \lambda n}, \quad\left(\tilde{q}=e^{-2 \pi / t}, t=\ell / r\right) .
\end{aligned}
$$

Because we quantized using $\sigma$ as time we obtain an answer in the closed string channel. We should perform a Poisson resummation to write the partition function in the open string channel,

$$
Z_{\lambda}=\sum_{m \in \mathbb{Z}} \frac{q^{(m+\lambda / 2)^{2}}}{\eta(q)}, \quad\left(q=e^{-\pi t}, t=\ell / r\right) .
$$

\section{FERMIONIC REPRESENTATION}

Having studied the orbifold theory at self-dual radius, we consider now other radii. At infinite radius, the renormalized bosonic theory may also be represented using a "free" fermionic picture [2,21]. We will take the liberty in this section of discussing this construction in detail. Although many aspects have been discussed in the literature, certain subtle points will be needed later in the paper when we apply the construction to orbifold theories.

The boundary interaction involves open string tachyonic vertex operators, $e^{ \pm i X / \sqrt{\alpha^{\prime}}}$. We should first study how this is represented in terms of the "doubled" chiral boson living on $\sigma \in[-r, r]$. When fermionizing we will quantize using $\tau$ as the time direction and the mode expansion of Sec. II, in contrast with the previous section. With $\hat{p}$ conjugate to $\hat{x}$, this vertex operator may be written:

$$
\begin{gathered}
e^{i k \hat{X}(\tau, 0) / \sqrt{\alpha^{\prime}}}=e^{i 2 k \hat{\phi}(\tau) / \sqrt{\alpha^{\prime}}} \\
e^{i k \hat{X}(\tau, r) / \sqrt{\alpha^{\prime}}}=e^{i 2 k \hat{\phi}(\tau+r) / \sqrt{\alpha^{\prime}}} e^{-i \pi \sqrt{\alpha^{\prime}} k\left(2 \hat{p}+k / \sqrt{\alpha^{\prime}}\right)} .
\end{gathered}
$$

In particular, when $k= \pm 1$ the vertex operator at $\sigma=r$ becomes

$$
e^{ \pm i \hat{X}(\tau, r) / \sqrt{\alpha^{\prime}}}=-e^{ \pm i 2 \hat{\phi}(\tau+r) / \sqrt{\alpha^{\prime}}} e^{\mp i 2 \pi \sqrt{\alpha^{\prime}} \hat{p}} .
$$

Thus the boundary interaction is

$$
\begin{aligned}
S_{\mathrm{int}}= & -\lambda \int_{\sigma=0} d s \cos \left(2 \phi(s) / \sqrt{\alpha^{\prime}}\right)+\frac{\tilde{\lambda}}{2} \\
& \times \int_{\sigma=r} d s\left(e^{i 2 \phi(s) / \sqrt{\alpha^{\prime}}} e^{-i 2 \pi \sqrt{\alpha^{\prime}} p}\right. \\
& \left.+e^{-i 2 \phi(s) / \sqrt{\alpha^{\prime}}} e^{i 2 \pi \sqrt{\alpha^{\prime}} p}\right) .
\end{aligned}
$$

\section{A. Fermionic action}

Here we want to find a fermionization appropriate for the boundary interaction. A convenient (but not unique) way to proceed is to mix in a second boson, $Y$. In [2] the second boson was viewed as auxiliary. However, in the context of string theory we may use one of the spatial directions as the second boson. It will be necessary to introduce cocycles in order for the algebraic properties to be faithfully reproduced. As $X$ was related to a chiral boson $\phi$, we may relate $Y$ to a chiral boson $\chi$. It is possible to take

$$
\begin{aligned}
& \psi_{1}=e^{i(\chi-\phi) / \sqrt{\alpha^{\prime}}} e^{i \pi a p} \equiv e^{i \phi_{1}} e^{i \pi a p}, \\
& \psi_{2}=e^{i(\chi+\phi) / \sqrt{\alpha^{\prime}}} e^{i \pi b p} \equiv e^{i \phi_{2}} e^{i \pi b p},
\end{aligned}
$$

where $a$ and $b$ are real parameters. As before $p$ is conjugate to $x$. We have chosen to write the fermion cocycles in terms of the $X$ zero modes so that the interaction is independent of the field, $Y$. We can introduce $p_{\phi}=2 p$, conjugate to $\phi_{0}$. Similarly we may introduce $p_{\chi}$, conjugate to $\chi_{0} \cdot \chi$ has a mode expansion similar to that of $\phi$.

The values of $a$ and $b$ may be constrained by demanding anticommutativity. This leads to the condition $\frac{b+a}{2 \sqrt{\alpha^{\prime}}} \in$ $2 \mathbb{Z}+1$. As in [2], we will choose $a=0$ and $b=-2 \sqrt{\alpha^{\prime}}$.

Recall that in the doubling we have

$$
\phi(\tau, r)=\phi(\tau,-r)+\pi \alpha^{\prime} p_{\phi} .
$$

Similarly,

$$
\chi(\tau, r)=\chi(\tau,-r)+\pi \alpha^{\prime} p_{\chi}
$$

Defining parameters $\zeta_{i}$, these periodicity conditions correspond to boundary conditions on the fermions

$$
\psi_{i}(r)=-e^{2 \pi i \zeta_{i}} \psi_{i}(-r) .
$$

The $\zeta_{i}$ correspond to the fractional (in units of $\sqrt{\alpha^{\prime}}$ ) parts of the momenta. From the fermionization map, we may relate the $\zeta_{i}$ to the momenta $p_{\phi}$ and $p_{\chi}(\bmod \mathbb{Z})$

$$
\zeta_{1}=\frac{\sqrt{\alpha^{\prime}}}{2}\left(p_{\chi}-p_{\phi}\right) ; \quad \zeta_{2}=\frac{\sqrt{\alpha^{\prime}}}{2}\left(p_{\chi}+p_{\phi}\right) .
$$

We will find it convenient to define $\zeta_{ \pm}=\frac{1}{2}\left(\zeta_{1} \pm \zeta_{2}\right)$. We then have

$$
\zeta_{+}=\frac{\sqrt{\alpha^{\prime}}}{2} p_{\chi}, \quad \zeta_{-}=-\frac{\sqrt{\alpha^{\prime}}}{2} p_{\phi} .
$$

Given the fermionization, the interaction becomes

$$
\begin{aligned}
S_{\mathrm{int}}= & -\frac{\lambda}{2} \int_{\Sigma}\left(\psi_{1}^{\dagger} \psi_{2} e^{i \pi \sqrt{\alpha^{\prime}} p_{\phi}}+\psi_{2}^{\dagger} \psi_{1} e^{-i \pi \sqrt{\alpha^{\prime}} p_{\phi}}\right) \delta(\sigma) \\
& +\frac{\tilde{\lambda}}{2} \int_{\Sigma}\left(\psi_{1}^{\dagger} \psi_{2}+\psi_{2}^{\dagger} \psi_{1}\right) \delta(\sigma-r) .
\end{aligned}
$$

Unfortunately, the first term of $L_{\text {int }}$ anticommutes with the fermion fields. However, it is possible to write an equivalent expression for the partition function that has a fermion 
number projection operator inserted (specifically, $(1+$ $\left.(-1)^{F_{1}+F_{2}}\right) / 2$ ), while adjusting the allowed values of $\zeta_{ \pm}$. In terms of this way of writing the partition function, the physical states have even fermion number, and we can with impunity modify the interaction by multiplying the first term in $L_{\text {int }}$ by $(-)^{F}$. These changes have no effect on the energy spectrum, but now the modified fermionic interaction commutes with the fermionic fields. This adjustment is required for the fermionization to make sense algebraically, and should be understood as part of the fermionization map.

Defining

$$
\Psi=\left(\begin{array}{l}
\psi_{1} \\
\psi_{2}
\end{array}\right),
$$

we may write the full Lagrangian as ${ }^{3}$

$$
\begin{aligned}
L= & \frac{1}{2 \pi} \int_{-\pi}^{\pi} d \sigma \Psi^{\dagger}\left(\partial_{\tau}-\partial_{\sigma}+i \mathbf{N}_{1} \delta(\sigma)\right. \\
& \left.-i \mathbf{N}_{2} \delta(\sigma-\pi)\right) \Psi,
\end{aligned}
$$

where we have defined matrices

$$
\mathbf{N}_{1}=\pi \lambda\left[\begin{array}{cc}
0 & w \\
\bar{w} & 0
\end{array}\right], \quad \mathbf{N}_{2}=\pi \tilde{\lambda}\left[\begin{array}{ll}
0 & 1 \\
1 & 0
\end{array}\right] .
$$

The factor $w$ is $e^{\sqrt{\alpha^{\prime}} \pi i p_{\phi}}(-)^{F}$. For fixed values of $\zeta_{+}, w$ takes the form $e^{-2 \pi i \zeta_{-}}(-)^{F}$.

If we wish to study the partition function of this theory, we need to diagonalize the Hamiltonian of the system. Following [2,21], we may Fourier transform in the $\tau$ direction

$$
\Psi(\tau, \sigma)=\int \frac{d \nu}{2 \pi} e^{-i \nu \tau} \tilde{\Psi}_{\nu}(\sigma) .
$$

Inserting this into the equation of motion gives constraints on the allowed values of $\nu$,

$$
\left(-i \nu-\partial_{\sigma}+i \mathbf{N}_{1} \delta(\sigma)-i \mathbf{N}_{2} \delta(\sigma-\pi)\right) \tilde{\Psi}_{\nu}(\sigma)=0 .
$$

Integrating, we find

$$
\tilde{\Psi}_{\nu}(\pi)=e^{-2 \pi i \nu} e^{-i \mathbf{N}_{2}} e^{i \mathbf{N}_{1}} \tilde{\Psi}_{\nu}(-\pi) .
$$

In this notation, the boundary conditions give also

$$
\tilde{\Psi}_{\nu}(\pi)=-e^{2 \pi i\left(\zeta_{+}+\sigma_{3} \zeta_{-}\right)} \tilde{\Psi}_{\nu}(-\pi) .
$$

To solve this equation we must demand that $\tilde{\Psi}_{\nu}(-\pi)$ be proportional to an eigenvector of

$$
\mathcal{N} \equiv e^{-i \mathbf{N}_{1}} e^{i \mathbf{N}_{2}} e^{2 \pi i \sigma_{3} \zeta_{-}}=\left(\begin{array}{cc}
\bar{w}(\cos (\pi \lambda) \cos (\pi \tilde{\lambda})+w \sin (\pi \lambda) \sin (\pi \tilde{\lambda})) & i w(\cos (\pi \lambda) \sin (\pi \tilde{\lambda})-w \sin (\pi \lambda) \cos (\pi \tilde{\lambda})) \\
i \bar{w}(\cos (\pi \lambda) \sin (\pi \tilde{\lambda})-\bar{w} \sin (\pi \lambda) \cos (\pi \tilde{\lambda})) & w(\cos (\pi \lambda) \cos (\pi \tilde{\lambda})+\bar{w} \sin (\pi \lambda) \sin (\pi \tilde{\lambda}))
\end{array}\right)
$$

The condition (38) and (39) also fixes $\nu$ up to integer shifts. The eigenvalues of $\mathcal{N}$ are $e^{ \pm 2 \pi i \alpha}$, where

$$
\begin{aligned}
\sin \pi \alpha= & \left(\sin ^{2}\left(\frac{\pi}{2}(\lambda-\tilde{\lambda})\right) \cos ^{2}\left(\pi \zeta_{-}\right)\right. \\
& \left.+\cos ^{2}\left(\frac{\pi}{2}(\lambda+\tilde{\lambda})\right) \sin ^{2}\left(\pi \zeta_{-}\right)\right)^{1 / 2}
\end{aligned}
$$

After renaming the values of $\nu$ which satisfy the equations of motion to $\omega$, we have the energy eigenvalues (for $\Psi$ ),

$$
\nu \equiv \omega_{n}^{ \pm}=n-\frac{1}{2}-\zeta_{+} \mp \alpha .
$$

We will define

$$
\Delta_{ \pm}=\zeta_{+} \pm \alpha\left(\zeta_{-}\right)
$$

We write the corresponding eigenvectors as

$$
u( \pm \alpha) \equiv\left(\begin{array}{l}
u_{1}^{( \pm)} \\
u_{2}^{( \pm)}
\end{array}\right) \text {. }
$$

\footnotetext{
${ }^{3}$ Henceforth we are setting $r=\pi$ in the range of $\sigma$.
}

\section{B. Solutions to fermionic EOM}

Next we present some notes on the structure of the Fock spaces. Our reason for discussing this in so much detail is that the energy eigenstate basis is not the natural one in the orbifold theory that we consider later in the paper. We may write the matrix which moves us between the 1-2 basis and the basis in which $\mathcal{N}$ is diagonal

$$
U=\left(\begin{array}{ll}
u_{1}^{(+)} & u_{2}^{(+)} \\
u_{1}^{(-)} & u_{2}^{(-)}
\end{array}\right) \equiv\left(\begin{array}{cc}
\eta \cos \theta & -\xi \sin \theta \\
\xi^{*} \sin \theta & \eta^{*} \cos \theta
\end{array}\right)
$$

When acting on the fermionic variables, we should enlarge this to

$$
\begin{gathered}
\hat{U}=\left(\begin{array}{cccc}
u_{1}^{(+)} & u_{2}^{(+)} & 0 & 0 \\
u_{1}^{(-)} & u_{2}^{(-)} & 0 & 0 \\
0 & 0 & \left(u_{1}^{(+)}\right)^{*} & \left(u_{2}^{(+)}\right)^{*} \\
0 & 0 & \left(u_{1}^{(-)}\right)^{*} & \left(u_{2}^{(-)}\right)^{*}
\end{array}\right) \\
\text { when acting on }\left(\begin{array}{c}
\psi_{1} \\
\psi_{2} \\
\psi_{1}^{\dagger} \\
\psi_{2}^{\dagger}
\end{array}\right) .
\end{gathered}
$$




\section{KAWAI, KESKI-VAKKURI, LEIGH, AND NOWLING}

With the vectors $u( \pm \alpha)$, we may write a general solution to the equation of motion,

$$
\begin{aligned}
\tilde{\Psi}_{\omega_{n}^{ \pm}}(\sigma)= & e^{-i \omega_{n}^{ \pm} \sigma} b_{n, \pm}\left(\left.1\right|_{[-\pi \ldots 0)}+\left.e^{i \mathbf{N}_{1}}\right|_{[0 \ldots \pi)}\right. \\
& \left.+\left.e^{-i \mathbf{N}_{2}} e^{i \mathbf{N}_{1}}\right|_{\sigma=\pi}\right) u( \pm \alpha) \\
\equiv & e^{-i \omega_{n}^{ \pm} \sigma} b_{n, \pm} u\left(\omega_{n}^{ \pm} ; \sigma\right) .
\end{aligned}
$$

The field $\Psi(\tau, \sigma)$ then has the mode expansion

$$
\Psi(\tau, \sigma)=\sum_{ \pm} \sum_{n \in \mathbb{Z}} e^{-i \omega_{n}^{ \pm}(\tau+\sigma)} b_{n, \pm} u\left(\omega_{n}^{ \pm} ; \sigma\right) .
$$

The $b_{n, \pm}$ are essentially the values of the excitations at the
PHYSICAL REVIEW D 73, 106003 (2006)

$\sigma=-\pi$ boundary. Similarly, the conjugate field is written

$$
\tilde{\Psi}_{\omega_{n}^{ \pm}}^{\dagger}(\sigma)=e^{+i \omega_{n}^{ \pm} \sigma} \bar{b}_{n, \pm} \bar{u}\left(\omega_{n}^{ \pm} ; \sigma\right) .
$$

By demanding that the pieces of $\Psi$ and $\Psi^{\dagger}$ which vanish as they approach the origin in the complex $z$ plane be proportional to creation operators, we can interpret

$$
b_{n, \pm}=\left\{\begin{array}{l}
\text { annihilation operator for } n>\frac{1}{2}+\Delta_{ \pm} \\
\text {creation operator for } n<\frac{1}{2}+\Delta_{ \pm}
\end{array}\right.
$$

with $\bar{b}_{n, \pm}$ as the conjugate operators with opposite action.

The normal ordered Hamiltonian is

$$
\begin{aligned}
: H: & =i \int d \sigma: \Psi^{\dagger} \partial_{\tau} \Psi:=\sum_{ \pm} \sum_{n \in \mathbb{Z}} \omega_{n}^{ \pm}: \bar{b}_{n, \pm} b_{n, \pm}: \equiv \sum_{ \pm}\left(\sum_{n \geq 1 / 2+\Delta_{ \pm}} \omega_{n}^{ \pm} \bar{b}_{n, \pm} b_{n, \pm}-\sum_{n<1 / 2+\Delta_{ \pm}} \omega_{n}^{ \pm} b_{n, \pm} \bar{b}_{n, \pm}\right) \\
& \equiv \sum_{ \pm}\left(\sum_{n \geq 1 / 2+\Delta_{ \pm}} \omega_{n}^{ \pm} \bar{b}_{n, \pm} b_{n, \pm}+\sum_{n>1 / 2-\Delta_{ \pm}} \bar{\omega}_{n}^{ \pm} b_{1-n, \pm} \bar{b}_{1-n, \pm}\right),
\end{aligned}
$$

with

$$
\omega_{n}^{ \pm}=n-1 / 2-\Delta_{ \pm}, \quad \bar{\omega}_{n}^{ \pm}=n-1 / 2+\Delta_{ \pm} .
$$

The vacuum of the Fock space, for given boundary conditions, then has the structure

$$
|\mathrm{vac}\rangle=\left[\prod_{n>1 / 2+\Delta_{+}}|n,+\rangle \prod_{\bar{n}>1 / 2-\Delta_{+}} \overline{|\bar{n},+\rangle} \prod_{n^{\prime}>1 / 2+\Delta_{-}}\left|n^{\prime},-\right\rangle \prod_{\bar{n}^{\prime}>1 / 2-\Delta_{-}} \overline{\left|\bar{n}^{\prime},-\right\rangle}\right]_{\left(\zeta_{+}, \zeta_{-}\right)} .
$$

Deformed Partition Function. -With the projection onto states of even total fermion number, (before integrating) the partition function takes the form:

$$
\begin{aligned}
Z\left(\zeta_{-}, \zeta_{+}\right) & =\frac{1}{2} \sum_{\epsilon= \pm} \prod_{\rho= \pm}\left(q^{\left[\left(\zeta_{+}+\rho \alpha\right)^{2} / 2\right]-(1 / 24)} \prod_{n}\left(1+\epsilon q^{n-1 / 2-\zeta_{+}-\rho \alpha}\right)\left(1+\epsilon q^{n-1 / 2+\zeta_{+}+\rho \alpha}\right)\right) \\
& =\sum_{m_{-}, m_{+} \in \mathbb{Z}} \frac{q^{\left(\zeta_{+}+m_{+}\right)^{2}+\left(\alpha+m_{-}\right)^{2}-(1 / 12)}}{\prod_{n}\left(1-q^{n}\right)^{2}} .
\end{aligned}
$$

and

$$
Z=\int_{0}^{1} d \zeta_{+} \int_{0}^{1} d \zeta_{-} Z\left(\zeta_{+}, \zeta_{-}\right)
$$

The ranges of the $\zeta_{ \pm}$integrations have been carefully chosen, given the fermion number projection, to cover the original open string momentum space once. The $Y$ system factors out (recall $p_{Y} \leftrightarrow \zeta_{+}$, and $\alpha$ is independent of $\left.\zeta_{+}\right)$:

$$
Z=Z_{Y} \cdot \int_{0}^{1} d \zeta_{-} \sum_{m_{-} \in \mathbb{Z}} \frac{q^{\left(\alpha+m_{-}\right)^{2}}}{\eta(q)}
$$

For comparison with other computations we can write this result in the closed string channel, via Poisson resumma- tion,

$$
Z=Z_{Y} \cdot \int_{0}^{1} d \zeta_{-} \frac{1}{\sqrt{2}} \sum_{\tilde{j}=0,1 / 2,1, \ldots} \chi_{\tilde{j}^{2}}^{V i r}\left(\tilde{q}^{2}\right) \chi_{\tilde{j}}^{S U(2)}\left(e^{4 \pi i \alpha J^{3}}\right) .
$$

\section{Boundary states at $\boldsymbol{R}=\infty$}

By observing the fermionized partition function, we can write a boundary state which correctly reproduces the open string partition function. Using the formula (9) we can write the partition function as an overlap of boundary states projected to infinite radius,

$$
\left\langle B\left(\tilde{\lambda} ; R=\sqrt{\alpha^{\prime}}\right)\left|P_{\infty} \Delta(\tilde{q}) P_{\infty}\right| B\left(\lambda ; R=\sqrt{\alpha^{\prime}}\right)\right\rangle,
$$


where

$$
\left.\left|B\left(\lambda ; R=\sqrt{\alpha^{\prime}}\right)\right\rangle=\sum_{j=0,1 / 2,1, \ldots} \sum_{m, n} \varphi^{j} \mathcal{D}_{m, n}^{(j)}\left(e^{2 \pi i \lambda J_{0}^{1}}\right)|j,-m, n\rangle\right\rangle .
$$

Note that there is a possible phase $\varphi$ here that is undetermined by the annulus computation; we will retain it for now. The projection to infinite radius is defined as [15]

$$
\left.P_{\infty}\left|B\left(\lambda ; R=\sqrt{\alpha^{\prime}}\right)\right\rangle=\int_{0}^{1} d \zeta_{-} \sum_{j=0,1 / 2,1, \ldots} \varphi^{j} \sum_{m, n} \mathcal{D}_{m, n}^{(j)}\left(e^{+2 \pi i \zeta_{-} J_{0}^{3}} e^{2 \pi i \lambda J_{0}^{1}} e^{+2 \pi i \zeta_{-} J_{0}^{3}}\right)|j,-m, n\rangle\right\rangle .
$$

Since $P_{\infty}$ is a projection operator, one may show that this boundary state is consistent with the form of $Z_{\lambda, \tilde{\lambda}}$. We may also show that this is equivalent to the standard expression [1]

$$
\left.P_{\infty}\left|B\left(\lambda ; R=\sqrt{\alpha^{\prime}}\right)\right\rangle=\sum_{j=0,1 / 2,1, \ldots} \varphi^{j} \sum_{m} \mathcal{D}_{-m, m}^{(j)}\left(e^{2 \pi i \lambda J_{0}^{1}}\right)|j, m, m\rangle\right\rangle
$$

where it is manifest that the winding modes have been projected out by the infinite radius limit.

Let us also check various limits of $\lambda$. First, take $\lambda=0$, which should give us the Neumann state:

$$
\left.P_{\infty}\left|B\left(0 ; R=\sqrt{\alpha^{\prime}}\right)\right\rangle=\sum_{j=0,1 / 2,1, \ldots} \varphi^{j}|j, 0,0\rangle\right\rangle,
$$

where we have used ${ }^{4} \mathcal{D}_{m, n}^{(j)}(\llbracket)=\delta_{m, n}$. This is the expected result.

For $\lambda=1 / 2$ :

$$
\left.P_{\infty}\left|B\left(1 / 2 ; R=\sqrt{\alpha^{\prime}}\right)\right\rangle=\sum_{j=0,1 / 2,1, \ldots} \sum_{m} \varphi^{j} \mathcal{D}_{-m, m}^{(j)}\left(e^{i \pi J_{0}^{1}}\right)|j, m, m\rangle\right\rangle
$$

and one finds $\mathcal{D}_{-m, m}^{(j)}\left(e^{i \pi J_{0}^{1}}\right)=e^{i \pi j}$. Thus we have

$$
\left.P_{\infty}\left|B\left(1 / 2 ; R=\sqrt{\alpha^{\prime}}\right)\right\rangle=\sum_{j=0,1 / 2,1, \ldots} \varphi^{j} e^{i \pi j} \sum_{m}|j, m, m\rangle\right\rangle .
$$

By rewriting this expression in oscillator variables, it becomes clear that it corresponds to an array of pointlike $D$-branes separated by a distance $2 \pi \sqrt{\alpha^{\prime}}$; that is, it is proportional to [4]

$$
\sum_{s \in \mathbb{Z}} \delta\left(x-(\pi / 2+2 \pi s) \sqrt{\alpha^{\prime}}\right),
$$

if we set the phase $\varphi$ to one. In fact, $\varphi$ simply corresponds to the freedom to translate the array of branes.

\footnotetext{
${ }^{4}$ In the notation used here, the general formula is

$\mathcal{D}_{m n}^{(j)}\left(\begin{array}{ll}a & b \\ c & d\end{array}\right)=\sum_{k} \frac{[(j+m) !(j-m) !(j+n) !(j-n) !]^{1 / 2}}{k !(j-m-k) !(j+n-k) !(m-n+k) !}$ $\times a^{j+n-k} b^{m-n+k} c^{k} d^{j-m-k}$.
}

\section{Projection to generic radii}

The fermionic calculation considered above is, strictly speaking, valid at infinite radius. The bosonic calculation is on the other hand valid at self-dual radius. With some care, we can in fact do the fermionic calculation at any (rational) radius. To our knowledge, this has not been described before from the open string point of view. In the boundary state formalism, there is a proposal [15,22], given by introducing suitable projection operators. By carefully considering the open string calculation, we will be able to explicitly display the meaning of these projection operators, and the limits of applicability of the fermionic picture.

To see that there is a potential problem in the fermionic theory, consider the fermion boundary conditions. Recall that $\zeta_{-}$is the fractional part of what was open string momentum in the bosonic picture. At finite radius, the open string momentum is quantized in units of $1 / R$, and thus one might expect that one could obtain the annulus amplitude by restricting the values of $\zeta_{-}$appropriately [2]. However, this only has limited applicability, to the case of integer radius (in units of $\sqrt{\alpha^{\prime}}$ ). One can easily show that this procedure fails for any other radius.

In the fermionic picture this can be seen from the boundary conditions: if the radius is not an integer, then $\zeta_{-}=1$ is not equivalent to $\zeta_{-}=0$, and this means that the 
fermionization does not make sense. This has roots in the fact that in the bosonic picture, the boundary operator $e^{i X / \sqrt{\alpha^{\prime}}}$ is not single-valued at generic radius, and thus the deformed theory does not exist. It is easy to repair this however, at least at rational radius, as we will now show.

Indeed, we may think of the finite radius theory as a shift orbifold, and define the theory by introducing a projection operator into open string correlators of the form

$$
P_{(R)}=\sum_{m \in \mathbb{Z}} e^{2 \pi i \hat{P} m R}
$$

where $\hat{P}$ is the momentum operator. In the undeformed theory, this implements the projection to finite radius correctly. In the deformed theory, the boundary operator

$$
S_{\lambda}=\frac{1}{2} \int_{\partial \Sigma} d s\left[\lambda e^{i X(s) / \sqrt{\alpha^{\prime}}}+\text { h.c. }\right]
$$

undergoes a transformation

$$
S_{\lambda} \rightarrow \frac{1}{2} \int_{\partial \Sigma} d s\left[\lambda e^{2 \pi i R / \sqrt{\alpha^{\prime}}} e^{i X(s) / \sqrt{\alpha^{\prime}}}+\text { h.c. }\right]
$$

under the shift $X \rightarrow X+2 \pi R$. Thus, if we insert $P_{(R)}$ into the path integral, it does not commute with the action precisely, and so the theory is not well-defined. It is easy to see how to repair this however; essentially, in the deformed boundary conformal field theory, we must include a nontrivial action in Chan-Paton corresponding to the shift. We may define a new theory by simply averaging the infinite radius theory over the values of $\lambda$ in the image of all possible shifts. If we write $R=(M / N) \sqrt{\alpha^{\prime}} \equiv r \sqrt{\alpha^{\prime}}$, then we would have for the annulus amplitude

$$
\mathcal{A}_{R ; \lambda, \tilde{\lambda}} \equiv \frac{1}{N^{2}} \sum_{n=0}^{N-1} \sum_{\tilde{n}=0}^{N-1} \operatorname{Tr}\left(P_{(R)} q^{L_{o}-1 / 24} e^{\left.S_{\lambda e^{2 \pi i n r}}+S_{\tilde{\lambda} e^{2 \pi i n} r}\right)} .\right.
$$

This theory has the interpretation of the Chan-Paton space for each boundary being extended to be $N$-component, each with a complex deformation parameter $\lambda e^{2 \pi i n r}$, for $n=0,1, \ldots, N-1$. Note that at integer radius $(N=1)$, this modification has no effect.

We emphasize that the expression (68) is an infinite radius calculation, expressed in the bosonic language. It is natural, because of the insertion, to evaluate it in momentum space. In this case, Eq. (68) differs from previous computations in two ways: first, there is a momentum dependent phase factor, and secondly, for any given $m, n$, $\tilde{n}$, there are effectively complex values of the boundary deformation parameters. Since we are at infinite radius, we may fermionize. In so doing, we find a generalization of the previous result: the important parameter $\alpha$ now takes the form

$$
\begin{aligned}
\sin ^{2} \pi \alpha= & \sin ^{2} \frac{\pi}{2}(|\lambda|-|\tilde{\lambda}|)+\sin ^{2} \pi \zeta_{-} \cos \pi|\lambda| \cos \pi|\tilde{\lambda}| \\
& +\sin ^{2} \pi(n-\tilde{n}) r \sin \pi|\lambda| \sin \pi|\tilde{\lambda}|
\end{aligned}
$$

depending in general on $|\lambda|, n,|\tilde{\lambda}|, \tilde{n}, \zeta_{-}$.

We recall that the open string momentum was split into an integer $\ell$ and $\zeta_{-} \in[0,1)$. Thus, we obtain

$$
\begin{aligned}
\mathcal{A}_{R ; \lambda, \tilde{\lambda}}= & \frac{1}{N^{2} \eta(q)} \sum_{m, \ell \in \mathbb{Z}} \sum_{n=0}^{N-1} \sum_{n=0}^{N-1} \int_{0}^{1} d \zeta_{-} e^{2 \pi i \zeta_{-} m r} e^{2 \pi i \ell m r} \\
& \times q^{(\ell+\alpha)^{2}}
\end{aligned}
$$

with $\alpha$ now given by Eq. (69), which is the generalization of previous results to complex couplings. Note that it is encoding the fact that $|\lambda|$ and $|\tilde{\lambda}|$ have been renormalized (in the fashion given by Ref. [1]) but the phases $n, \tilde{n}$ are essentially not renormalized..$^{5}$ Thus the result is a function of $|\lambda|, n ;|\tilde{\lambda}|, \tilde{n}$ but not just of $\lambda, \tilde{\lambda}$.

It is important to realize that what we have done here is to resolve the classical problem of finite radius. In going to the fermionic representation however, there is a potential quantum problem as well - that is, the fermionic measure may not be well defined in the presence of the projection operator. Indeed, the translation operator corresponds precisely to a chiral $\mathbb{Z}_{2 N}$ transformation on the fermions, and thus the measure is not invariant, transforming by a $\mathbb{Z}_{N}$ phase. This may be repaired by introducing an array of Wilson lines, as

$$
\begin{aligned}
\mathcal{A}_{R ; \lambda, \tilde{\lambda}}^{\text {fermionic }}= & \frac{1}{N^{2} \eta(q)} \sum_{m, \ell \in \mathbb{Z}} \sum_{n=0}^{N-1} \sum_{\tilde{n}=0}^{N-1} \int_{0}^{1} d \zeta_{-} e^{2 \pi i \zeta_{-} m r} e^{2 \pi i \ell m r} \\
& \times q^{(\ell+\alpha)^{2}} \sum_{k=0}^{N-1} e^{-2 \pi i m k / N}
\end{aligned}
$$

The idea is that the action of the projection operator on the fermionic measure leads to a $\mathbb{Z}_{N}$ phase, which can be absorbed by a shift to another Wilson line. Thus, we obtain a well-defined fermionic theory by summing over such Wilson lines. It is important to note here that what is being said is that consistent fermionic theories can be defined only if we include the array of Wilson lines. The bosonic theory exists in the absence of the Wilson line array, but we have no way to compute in the deformed theory, away from self-dual radius. Note further that the Wilson line array appears only for fractional radii (i.e., $N \neq 1$ ); the integer radius cases work just fine without it.

Now what the array of Wilson lines does is force $m=$ $N s$, for $s \in \mathbb{Z}$. We then obtain

\footnotetext{
${ }^{5}$ The reason for this dichotomy is that the renormalization comes from the collision of $J_{+}$and $J_{-}$insertions, each of which is accompanied by $|\lambda|$, but opposite phases. Thus the powers of $|\lambda|$ build up, but the phases tend to cancel and do not renormalize.
} 


$$
\begin{aligned}
\mathcal{A}_{R ; \lambda, \tilde{\lambda}}^{\text {fermionic }}= & \frac{1}{N^{2} \eta(q)} \sum_{s, \ell \in \mathbb{Z}} \sum_{n=0}^{N-1} \sum_{n=0}^{N-1} \int_{0}^{1} d \zeta_{-} e^{2 \pi i \zeta_{-} M s} q^{(\ell+\alpha)^{2}} \\
= & \frac{1}{M N^{2} \eta(q)} \sum_{\ell \in \mathbb{Z}} \sum_{n=0}^{N-1} \sum_{n=0}^{N-1} \int_{0}^{1} d \zeta_{-} \\
& \times \sum_{k^{\prime} \in \mathbb{Z}} \delta\left(\zeta_{-}-k^{\prime} / M\right) q^{(\ell+\alpha)^{2}} \\
= & \frac{1}{M N^{2} \eta(q)} \sum_{\ell \in \mathbb{Z}} \sum_{n, \tilde{n}=0}^{N-1} \sum_{k^{\prime}=0}^{M-1} q^{\left(\ell+\alpha\left(\zeta_{-}=k^{\prime} / M\right)\right)^{2}}
\end{aligned}
$$

For later use, we note that this can be manipulated into the form

$$
\frac{1}{M N \eta(q)} \sum_{\ell \in \mathbb{Z}} \sum_{k=0}^{N-1} \sum_{k^{\prime}=0}^{M-1} q^{\left(\ell+\beta\left(\zeta_{-}=k^{\prime} / M\right)\right)^{2}}
$$

where

$$
\begin{aligned}
\cos 2 \pi \beta= & \cos \pi|\lambda| \cos \pi|\tilde{\lambda}| \cos 2 \pi \zeta_{-} \\
& +\sin \pi|\lambda| \sin \pi|\tilde{\lambda}| \cos 2 \pi k / N
\end{aligned}
$$

This formula is a direct consequence of (69).

Let us consider a few special cases. First, the NN amplitude should be recovered by taking $\lambda, \tilde{\lambda} \rightarrow 0$. In this case, $\alpha=\zeta_{-}$, independent of $n, \tilde{n}$, and so we find

$$
\frac{1}{M \eta(q)} \sum_{\ell \in \mathbb{Z}} \sum_{k^{\prime}=0}^{M-1} q^{\left(\ell+k^{\prime} / M\right)^{2}}=\frac{1}{M \eta(q)} \sum_{\ell \in \mathbb{Z}} q^{\ell^{2} / M^{2}}
$$

which may be recognized as the NN amplitude at radius $R$, with the array of Wilson lines. Note also that

$$
\mathcal{A}_{R ; 1,0}=\frac{1}{M \eta(q)} \sum_{\ell \in \mathbb{Z}} q^{(\ell / M+1 / 2)^{2}} .
$$

Next let us consider the ND case, obtained by $\lambda=1 / 2$, $\tilde{\lambda}=0$. Here, we obtain $\alpha=1 / 4$, independent of $n, \tilde{n}$, as well as $\zeta_{-}$and thus

$$
\mathcal{A}_{R ; 1 / 2,0}=\frac{1}{\eta(q)} \sum_{\ell \in \mathbb{Z}} q^{(\ell+1 / 4)^{2}} .
$$

Finally, consider the DD case, obtained via $\lambda=\tilde{\lambda}=$ $1 / 2$, whence $\alpha=(n-\tilde{n}) r$. Thus, we find

$$
\mathcal{A}_{R ; 1 / 2,1 / 2}=\frac{1}{N^{2} \eta(q)} \sum_{\ell \in \mathbb{Z}} \sum_{n, \tilde{n}=0}^{N-1} q^{(\ell+(n-\tilde{n}) r)^{2}}
$$

It is possible to show that the sum over $n, \tilde{n}$ can be written as

$$
\mathcal{A}_{R ; 1 / 2,1 / 2}=\frac{1}{N \eta(q)} \sum_{\ell \in \mathbb{Z}} \sum_{k=-j}^{j} q^{(N \ell+k M)^{2} / N^{2}}
$$

where $j=[[N / 2]]$. This is

$$
\mathcal{A}_{R ; 1 / 2,1 / 2}=\frac{1}{N \eta(q)} \sum_{\ell \in \mathbb{Z}} q^{\ell^{2} / N^{2}}
$$

which is the correct result for an array of $M D$-branes, located at integer multiples of $\sqrt{\alpha^{\prime}} / N$. This is the expected result [4], with branes located at extrema of the boundary potential. It is clear that this array of $D$-branes is $T$-dual to the array of Wilson lines at $\lambda=\tilde{\lambda}=0$. For generic values of $\lambda, \tilde{\lambda}$, we interpolate smoothly between the two arrays, again as should be expected. The absence of the fermionic anomaly mentioned above corresponds to the recovery of $T$-duality in the annulus amplitude.

\section{Boundary states at radius $r$}

These finite radius annulus amplitudes may be transformed into the closed string channel. The result is consistent with boundary states given (from closed channel reasoning) by Gaberdiel and Recknagel [15]. They are written via projection operators acting on the self-dual radius result (here we have made a requisite translation into our conventions)

$$
\begin{aligned}
|B(\lambda ; R)\rangle & \left.=P_{M}^{+} P_{N}^{-} \sum_{j_{0,1 / 2} \ldots} \sum_{m, \tilde{m}=-j}^{j} \varphi^{j} \mathcal{D}_{m \tilde{m}}^{j}\left(e^{2 \pi i \lambda J^{1}}\right)|j,-m, \tilde{m}\rangle\right\rangle \\
& \left.\equiv \frac{1}{\sqrt{M N}} \sum_{\ell=0}^{M-1} \sum_{k=0}^{N-1} \sum_{j_{0,1 / 2, \ldots}} \sum_{m, \tilde{m}=-j}^{j} \varphi^{j} \mathcal{D}_{m \tilde{m}}^{j}\left(e^{2 \pi i((\ell / M)+(k / N)) J^{3}} e^{2 \pi i \lambda J^{1}} e^{2 \pi i((\ell / M)-(k / N)) J^{3}}\right)|j,-m, \tilde{m}\rangle\right\rangle .
\end{aligned}
$$

Using this boundary state, one obtains the following for the open string partition function,

$$
\frac{1}{M N} \sum_{\ell=0}^{M-1} \sum_{k=0}^{N-1} \sum_{n \in \mathbb{Z}} \frac{q^{(n+\beta(\lambda, \tilde{\lambda} ; \ell, k))^{2}}}{\eta(q)}
$$

Here $\beta$ satisfies 


$$
2 \cos (2 \pi \beta(\lambda, \tilde{\lambda} ; \ell, k)) \equiv \operatorname{Tr}_{1 / 2}\left(e^{-2 \pi i \tilde{\lambda} J^{1}} e^{2 \pi i((\ell / M)+(k / N)) J^{3}} e^{2 \pi i \lambda J^{1}} e^{2 \pi i((\ell / M)-(k / N)) J^{3}}\right) .
$$

One may show that the set of values of $\beta$ is equivalent to the set of $\alpha$ 's in Eq. (72). This result is equivalent to the open string result, as long as the array of Wilson lines is included. Note that this is important, because the boundary states proposed transform in a simple way under $T$-duality. Without the Wilson lines, $T$-duality is not implemented by flowing from $\lambda=0$ to $\lambda=1 / 2$.

\section{THE $\mathbb{Z}_{2}$ ORBIFOLD}

We will now consider the orbifold action in the boundary conformal field theory (14). For the annulus computation, this is implemented by including the orbifold projection operator $\frac{1}{2}(1+g)$ in the path integral. The first term (proportional to 1) is equivalent, up to the factor of 2 , to the results given above. On a self-dual circle we may derive the effect of inserting $g$ through adsorption methods (see Ref. [12] for the construction in the undeformed theory). Because the states are classified by current algebra and we know the orbifold action on the algebra, it is straightforward to compute the $g$-inserted trace at self-dual radius. As in the previous sections, to discuss more general radii we must fermionize the theory. When fermionizing we will consider two possible orbifold actions: either $g$ acts only the $X$ field (" $c=1$ orbifold") or it acts on both $X$ and $Y$ (" $c=2$ orbifold"). In either case, we should be able to disentangle the ( $g$-inserted) partition functions of the $X$ and $Y$ systems. The consistency of these three routes is strong evidence that we have correctly orbifolded the deformed boson.

In the orbifold theory, there are a variety of open string annulus calculations that we can do, depending on the details of Chan-Paton factors, as we have discussed briefly above. We will consider these details in Ref. [8] and here simply concentrate on the calculation of the annulus diagram with orbifold insertion. This will be the basic building block needed to construct fractional boundary states.

\section{A. Self-dual radius and adsorption}

The orbifold is obtained by defining $\mathbb{Z}_{2}$ as $X \mapsto-X$, or in terms of the current,

$$
J^{1} \rightarrow J^{1} ; \quad J^{2} \rightarrow-J^{2} ; \quad J^{3} \rightarrow-J^{3} .
$$

At self-dual radius, it is convenient to organize the calculation entirely in terms of the $\widehat{S U(2)})_{1}$ modules. Since the deformation is in the direction of $J^{1}$ rather than $J^{3}$, it is more convenient to work in the $S U(2)$ basis where $J_{0}^{1}$ is diagonal, as we described in Sec. III. The orbifold action then switches the sign of the ladder operators. The $g$-inserted partition function is (see the appendix for a detailed analysis)

$$
\operatorname{Tr} g q^{L_{0}-1 / 24}=\frac{1}{\eta(q)} \sum_{n \in \mathbb{Z}}(-1)^{n} q^{(n+\lambda / 2)^{2}} .
$$

For other radii, we now turn to the fermionic description.

\section{B. Fermionic description of the orbifold}

Let us discuss the $\mathbb{Z}_{2}$ orbifold of the deformed theory. After detailed computations, the two orbifold actions may be determined consistently to be as given in the following table.

\begin{tabular}{ll}
\hline \hline$c=2$ Orb & $c=1$ Orb \\
\hline$X \rightarrow-X$ & $X \rightarrow-X$ \\
$Y \rightarrow-Y$ & $Y \rightarrow Y$ \\
$\psi_{1} \rightarrow \psi_{1}^{\dagger}$ & $\psi_{1} \rightarrow i \psi_{2} e^{2 \pi i \zeta_{-}}$ \\
$\psi_{2} \rightarrow-\psi_{2}^{\dagger}$ & $\psi_{2} \rightarrow i \psi_{1} e^{2 \pi i \zeta_{-}}$ \\
$\psi_{1}^{\dagger} \rightarrow \psi_{1}$ & $\psi_{1}^{\dagger} \rightarrow-i \psi_{2}^{\dagger} e^{-2 \pi i \zeta_{-}}$ \\
$\psi_{2}^{\dagger} \rightarrow-\psi_{2}$ & $\psi_{2}^{\dagger} \rightarrow-i \psi_{1}^{\dagger} e^{-2 \pi i \zeta_{-}}$ \\
\hline \hline
\end{tabular}

The phases that appear in the table may look strange; in particular, it might appear that $g_{1}$ does not square to one. However, the table refers to the action on single fermions, which do not by themselves create physical states (recall the fermion number projection). The phases in the table are fixed by the requirement that the $S U(2)_{X}$ current algebra as well as other fermion bilinears transform in a sensible way under the orbifold actions. In particular, $g$ does square to one on all physical states, in each case.

In order to proceed we need to rephrase the orbifold as an action in the \pm basis rather than the 1-2 basis. This is accomplished by making a similarity transformation on the orbifold generator, $\hat{U} g \hat{U}^{\dagger}$. The action of $g_{1}$ on the $\psi-\psi^{\dagger}$ and \pm labels of the field is given by:

$$
\hat{U} g_{1} \hat{U}^{-1}=\left(\begin{array}{cc}
i G_{1} & 0 \\
0 & -i G_{1}^{*}
\end{array}\right)
$$

where

$$
G_{1}=e^{2 \pi i \zeta_{-}} U g U^{-1},
$$

with $g$ from the table,

$$
g=\left(\begin{array}{ll}
0 & 1 \\
1 & 0
\end{array}\right),
$$

and $U$ was given in a preceding section. The action of $g_{2}$ on the $\psi-\psi^{\dagger}$ and \pm labels of the field is given by:

$$
\hat{U} g_{2} \hat{U}^{-1}=\left(\begin{array}{cc}
0 & G_{2} \\
G_{2}^{*} & 0
\end{array}\right)
$$

where

$$
G_{2}=U g U^{-1}
$$


and from the table,

$$
g=\left(\begin{array}{cc}
1 & 0 \\
0 & -1
\end{array}\right)
$$

Having determined how the orbifold acts on the fields of the theory, it remains to determine the action on the ground state. Fortunately, this is facilitated by the fact that we knew how the orbifold acted upon the momenta of the bosonic theory. Consistency demands that $g$ acts on the $\zeta_{-}$and $\zeta_{+}$as it did on the $X$ and $Y$ momenta, respectively. The orbifold action on the interacting ground state is:

$$
\begin{aligned}
& g_{1} \cdot\left[|n,+\rangle \otimes \overline{|\bar{n},+\rangle} \otimes\left|n^{\prime},-\right\rangle \otimes \overline{\left|\bar{n}^{\prime},-\right\rangle}\right]_{\left(\zeta_{+}, \zeta_{-}\right)} \\
& \quad=\left[|n,+\rangle \otimes \overline{|\bar{n},+\rangle} \otimes\left|n^{\prime},-\right\rangle \otimes \overline{\left|\bar{n}^{\prime},-\right\rangle}\right]_{\left(\zeta_{+},-\zeta_{-}\right)} \\
& g_{2} \cdot\left[|n,+\rangle \otimes \overline{|\bar{n},+\rangle} \otimes\left|n^{\prime},-\right\rangle \otimes \overline{\left|\bar{n}^{\prime},-\right\rangle}\right]_{\left(\zeta_{+}, \zeta_{-}\right)} \\
& \quad=\left[|n,+\rangle \otimes \overline{|\bar{n},+\rangle} \otimes\left|n^{\prime},-\right\rangle \otimes \overline{\left|\bar{n}^{\prime},-\right\rangle}\right]_{\left(-\zeta_{+},-\zeta_{-}\right)}
\end{aligned}
$$

We see that $g_{1}$ effectively flips the sign of $\zeta_{-}$and $g_{2}$ flips the sign of both $\zeta_{+}$and $\zeta_{-}$inside the vacuum states.

\section{Orbifold-inserted traces}

Because the orbifold action mixes all of the oscillators, at each value of $n$ there is the possibility for many terms to contribute. The orbifold actions on the vacuum states limit the values of $\zeta_{ \pm}$which contribute to the $g$-inserted partition functions - that is, there will be $\delta$-functions which restrict to the fixed points of the orbifold action. As before, we use the same fermion insertion $\left(1+(-1)^{F_{T}}\right) / 2$ and integration measure $\int_{0}^{1} d \zeta_{+} \int_{0}^{1} d \zeta_{-}$.

$c=1$ Orbifold. - Since $\zeta_{+} \mapsto \zeta_{+}$and $\zeta_{-} \mapsto-\zeta_{-}$under the orbifold action, there is a fixed line at $\zeta_{-}=0$. Here, $\alpha=(\lambda-\tilde{\lambda}) / 2(\bmod 1)$, and

$$
U=\left(\begin{array}{cc}
\frac{1}{\sqrt{2}} & -\frac{1}{\sqrt{2}} \\
\frac{1}{\sqrt{2}} & \frac{1}{\sqrt{2}}
\end{array}\right)
$$

and thus the orbifold action on single-fermion states is determined by

$$
\begin{aligned}
\hat{U} g_{1} \hat{U}^{\dagger} & =\left(\begin{array}{cc}
i G & 0 \\
0 & -i G^{*}
\end{array}\right) \\
& =\left(\begin{array}{cccc}
-i e^{2 \pi i \zeta_{-}} & 0 & 0 & 0 \\
0 & i e^{2 \pi i \zeta_{-}} & 0 & 0 \\
0 & 0 & i e^{-2 \pi i \zeta_{-}} & 0 \\
0 & 0 & 0 & -i e^{-2 \pi i \zeta_{-}}
\end{array}\right) .
\end{aligned}
$$

Note that the factors of $i$ that appear here are required by consistency (although they did not appear "geometrically"). As we discussed above, it looks as if $g_{1}^{2}=-1$; however, this is acting on single-fermion states-since there are no such physical states, we can allow such an action. It must act as $g_{1}^{2}=+1$ on all double fermion states however. We can see that we need the factors of $i$ by examining operators like $\psi_{+} \psi_{-}$, which consists of $Y$ only, and thus should be orbifold invariant.

There is also a fixed line at $\zeta_{-}=1 / 2$, because $\zeta$ 's are defined $\bmod 1$. Here, $\alpha=(\lambda+\tilde{\lambda}-1) / 2(\bmod 1)$ and $U$ is the same as above.

The $g_{1}$ trace takes the form $\int_{0}^{1} d \zeta_{+} \int_{0}^{1} d \zeta_{-}\left[\delta\left(\zeta_{-}\right)+\right.$ $\left.\delta\left(\zeta_{-}-1 / 2\right)\right]$ times

$$
\begin{aligned}
& q^{\zeta_{+}^{2}+\alpha^{2}-1 / 12} \frac{1}{2} \sum_{ \pm} \prod_{n=1}^{\infty}\left(1 \mp i e^{2 \pi i \zeta_{-}} q^{\omega_{n}^{+}}\right)\left(1 \pm i e^{2 \pi i \zeta_{-}} q^{\omega_{n}^{-}}\right)(1 \\
& \left.\quad \pm i e^{-2 \pi i \zeta_{-}} q^{\bar{\omega}_{n}^{+}}\right)\left(1 \mp i e^{-2 \pi i \zeta_{-}} q^{\bar{\omega}_{n}^{-}}\right) .
\end{aligned}
$$

The sum $\sum_{ \pm}$is the fermion number projection. This result can be written in terms of a product of $\theta$-functions; using the sum representation for the $\theta$-functions, we then find

$$
\begin{aligned}
& \frac{q^{\zeta_{+}^{2}+\alpha^{2}}}{\eta^{2}(q)} \sum_{n, m \in \mathbb{Z}} q^{n^{2} / 2} q^{m^{2} / 2}\left(i e^{2 \pi i \zeta_{-}} q^{-\zeta_{+}-\alpha}\right)^{n}\left(i e^{2 \pi i \zeta_{-}} q^{-\zeta_{+}+\alpha}\right)^{m} \\
& \quad \times\left[\frac{(-1)^{n}+(-1)^{m}}{2}\right]
\end{aligned}
$$

Defining $m_{ \pm}=(n \pm m) / 2$, we find

$$
\begin{aligned}
Z_{\lambda, \tilde{\lambda}}^{g_{1}} & =\int d \zeta_{+} \sum_{\zeta_{-}=0,1 / 2} \frac{q^{\zeta_{+}^{2}+\alpha^{2}}}{\eta^{2}(q)} \sum_{m_{+}, m_{-} \in \mathbb{Z}} q^{m_{+}^{2}} q^{m_{-}^{2}} e^{4 \pi i \zeta_{-} m_{+}} q^{-2 \zeta_{+} m_{+}} q^{-2 \alpha m_{-}}(-1)^{m_{-}} \\
& =\left(\frac{1}{\eta(q)} \int_{0}^{1} d \zeta_{+} \sum_{m_{+} \in \mathbb{Z}} q^{\left(m_{+}+\zeta_{+}\right)^{2}}\right) \cdot\left(\sum_{\zeta_{-}=0,1 / 2} \frac{1}{\eta(q)} \sum_{m_{-} \in \mathbb{Z}} q^{\left(m_{-}+\alpha\right)^{2}}(-1)^{m_{-}}\right)
\end{aligned}
$$

This is to be summed over the two values of $\zeta_{-}$; for both of those values, the $\zeta_{-}$-dependent term in the $m_{+}$sum equals unity, and we have dropped it. So, we see that the $g_{1}$-inserted partion function decouples nicely into $X$ and $Y$. The integral over $\zeta_{+}$combines with the $m_{+}$sum to give the partition function of the free boson $Y$ while the $m_{-}$sum is the twisted partition function of $X$.

$c=2$ Orbifold. - Because both $\zeta_{ \pm}$are set to zero modulo periodicities in the $c=2$ orbifold, we only get contributions from the points $(0,0)$ and $(1 / 2,1 / 2)$. The action 
of the orbifold on the fermion fields is given by:

$$
\hat{U} g_{2} \hat{U}^{\dagger}=\left(\begin{array}{cccc}
0 & 0 & 0 & 1 \\
0 & 0 & 1 & 0 \\
0 & 1 & 0 & 0 \\
1 & 0 & 0 & 0
\end{array}\right) .
$$

The diagonal combinations of states are

$$
|\mathrm{vac}\rangle, \psi_{-}^{\dagger} \psi_{+}|\mathrm{vac}\rangle, \psi_{+}^{\dagger} \psi_{-}|\mathrm{vac}\rangle, \psi_{-}^{\dagger} \psi_{+} \psi_{+}^{\dagger} \psi_{-}|\mathrm{vac}\rangle
$$

For the two points, we find

$$
\begin{aligned}
& \text { at } \zeta_{\mp}=0: \alpha(0)=\frac{\lambda-\tilde{\lambda}}{2}, \quad \text { Casimir energy }=\left(\frac{\lambda-\tilde{\lambda}}{2}\right)^{2}-\frac{1}{12} \\
& \text { at } \zeta_{\mp}=1 / 2: \alpha(1 / 2)=\frac{\lambda+\tilde{\lambda}-1}{2}, \quad \text { Casimir energy }=\left(\frac{\lambda+\tilde{\lambda}-1}{2}\right)^{2}-\frac{1}{12} .
\end{aligned}
$$

The trace, with $g_{2}$ inserted, becomes:

$$
\begin{aligned}
Z_{\lambda, \tilde{\lambda}}^{g_{2}} & =\sum_{\left(\zeta_{-}, \zeta_{+}\right)=(0,0),(1 / 2,1 / 2)} q^{\alpha^{2}-1 / 12} \prod_{n}\left(1-\left(q^{2}\right)^{n-1 / 2+\alpha}\right)\left(1-\left(q^{2}\right)^{n-1 / 2-\alpha}\right) \\
& =\left(\frac{q^{-1 / 24}}{\prod_{n}\left(1+q^{n}\right)}\right) \cdot\left(\sum_{\zeta_{-}=0,1 / 2} \frac{1}{\eta(q)} \sum_{n \in \mathbb{Z}}(-)^{n} q^{(n+\alpha)^{2}}\right) .
\end{aligned}
$$

We have split the trace into separate contributions from $\left(\zeta_{-}, \zeta_{+}\right)=(0,0)$ and $(1 / 2,1 / 2)$. The minus sign in the factors is due to the anticommutivity of the fermion fields. Note that the powers of $q$ here are all independent of $\zeta_{+}$, an important feature. The insertion of $g_{2}$ restricts the trace to be over states of even fermion number, implying that the total fermion number projection operator acts as the identity in the presence of $g_{2}$. Again, the $g$-inserted partition function has factorized into contributions from $X$ and $Y$.

\section{Summary}

At the self-dual radius, one finds

$$
Z_{\lambda, \tilde{\lambda}}^{g}=\frac{1}{\eta(q)} \sum_{n \in \mathbb{Z}}(-)^{n} q^{(n+(\lambda-\tilde{\lambda}) / 2)^{2}}
$$

using adsorption methods. For infinite radius, we may decouple the $Y$-system from the result of fermionization to obtain

$$
Z_{1}=\frac{1}{\eta(q)} \int_{0}^{1} d \zeta_{-} \sum_{m \in \mathbb{Z}} q^{\left(m+\alpha\left(\zeta_{-}\right)\right)^{2}}
$$

and

$$
Z_{g}=\frac{1}{\eta(q)} \sum_{\zeta_{-}=0,1 / 2} \sum_{m \in \mathbb{Z}}(-1)^{m} q^{\left(m+\alpha\left(\zeta_{-}\right)\right)^{2}}
$$

Here,

$$
\begin{aligned}
\sin \pi \alpha= & \left(\sin ^{2}\left(\frac{\pi}{2}(\lambda-\tilde{\lambda})\right) \cos ^{2}\left(\pi \zeta_{-}\right)\right. \\
& \left.+\cos ^{2}\left(\frac{\pi}{2}(\lambda+\tilde{\lambda})\right) \sin ^{2}\left(\pi \zeta_{-}\right)\right)^{1 / 2} .
\end{aligned}
$$

Notice that the $\zeta_{-}=0$ term reproduces the self-dual radius result. This is correct, since at self-dual radius half- integer momentum $\left(\zeta_{-}=1 / 2\right)$ is not present. It is a confirmation of our methods that the contributions at $\zeta_{-}=0$ to $Z_{\lambda, \tilde{\lambda}}^{g}$ obtained from fermionization match the result of the self-dual radius adsorption methods.

Also recall that for $Z_{1}$, we can write the result in terms of Virasoro and $S U(2)$ characters of the closed string channel

$$
\begin{aligned}
Z_{1} & =\frac{1}{\sqrt{2}} \frac{1}{\eta\left(\tilde{q}^{2}\right)} \int_{0}^{1} d \zeta_{-} \sum_{m \in \mathbb{Z}}\left(\tilde{q}^{2}\right)^{m^{2} / 4} e^{2 \pi i \alpha\left(\zeta_{-}\right) m} \\
& =\frac{1}{\sqrt{2}} \sum_{j=0,1 / 2,1, \ldots} \chi_{j^{2}}^{V i r}\left(\tilde{q}^{2}\right) \int_{0}^{1} d \zeta_{-} \chi_{j}^{S U(2)}\left(e^{4 \pi i \alpha\left(\zeta_{-}\right) J^{3}}\right) .
\end{aligned}
$$

The corresponding result for $Z_{g}$ follows from Poisson resummation

$$
Z_{g}=\frac{1}{\sqrt{2}} \frac{1}{\eta\left(\tilde{q}^{2}\right)} \sum_{\zeta_{-}=0,1 / 2} \sum_{m \in \mathbb{Z}}\left(\tilde{q}^{2}\right)^{(m-1 / 2)^{2} / 4} e^{2 \pi i \alpha\left(\zeta_{-}\right)(m-1 / 2)} .
$$

Other radii may be obtained by suitable projection operators, as discussed in Sec. IV D. A treatment of all possible boundary states of the orbifold theory appears in Ref. [8].

\section{CONCLUSIONS}

In this paper, we have considered exactly marginal boundary deformations of the $c=1$ theory through oneloop computations directly in the open string channel. The partition function at generic radius was constructed through a fermionization technique and shown to coincide with expected boundary states. In doing so, it was necessary to carefully define the fermionic theory so as to be consistent with the projection to finite radius. We have also carefully constructed the orbifold $\mathbb{R}^{d} / \mathbb{Z}_{2}$ theory in the 


\section{ROLLING TACHYON BOUNDARY CONFORMAL FIELD ...}

fermionic parameterization and computed the corresponding twisted partition function. Further results and applications of our results will appear in Ref. [8].

\section{ACKNOWLEDGMENTS}

E. K. V thanks UCLAs IPAM and the organizers of the Conformal Field Theory 2nd Reunion Conference, and the organizers of the Workshop on Gravitational Aspects of String Theory at Fields Institute for hospitality while this work was in progress. S. K. would like to thank Matthias Gaberdiel, Andreas Recknagel, and Masaki Oshikawa for discussions, and also the organizers of the July 2005
PHYSICAL REVIEW D 73, 106003 (2006)

London Mathematical Society Durham Symposium on "Geometry, Conformal Field Theory and String Theory." E. K. V. was in part supported by the Academy of Finland. S. K. was in part supported by JSPS. R. G. L. and S. N. have support from the US Department of Energy under Contract No. DE-FG02-91ER40709.

\section{APPENDIX}

For convenience, we present more details of the $S U(2)$ module calculations of Secs. III and VA here. The multiplicities of the $j=0 S U(2)$ module can be found in [14], Fig. 15.1. By direct computation, we obtain

$$
\begin{aligned}
\operatorname{tr} q^{L_{0}-1 / 24} & =\left(1 \cdot q^{0}\right)+(1+1+1) \cdot q^{1}+(1+2+1) \cdot q^{2}+\ldots \\
& =\left(1+q+2 q^{2}+\ldots\right)+2 q\left(1+q+2 q^{2}+\ldots\right)+2 q^{4}(1+q+\ldots)+\ldots=\frac{1}{\eta(q)} \sum_{n \in \mathbb{Z}} q^{n^{2}}
\end{aligned}
$$

In the basis where $J^{1}$ is diagonal, $g$ inverts the sign of ladder operators (or rather, $g$ anticommutes with $J^{ \pm}$). Thus, inserting $g$ we obtain $g=(-1)^{m}$ and so

$$
\begin{aligned}
\operatorname{tr} g q^{L_{0}-1 / 24} & =\left(1 \cdot q^{0}\right)+(-1+1-1) \cdot q^{1}+(-1+2-1) \cdot q^{2}+\ldots \\
& =\left(1+q+2 q^{2}+\ldots\right)-2 q\left(1+q+2 q^{2}+\ldots\right)+2 q^{4}(1+q+\ldots)+\ldots=\frac{1}{\eta(q)} \sum_{n \in \mathbb{Z}}(-1)^{n} q^{n^{2}} .
\end{aligned}
$$

With the deformation, the Hamiltonian is shifted to $L_{o}=(m+\lambda / 2)^{2}+\left(N-m^{2}\right)=N+\lambda m+\lambda^{2} / 4$. The $j=0$ contribution is

$$
\begin{aligned}
\operatorname{tr} q^{L_{0}-1 / 24} & =q^{\lambda^{2} / 4}\left\{(1) \cdot q^{0}+\left(q^{\lambda}+1+q^{-\lambda}\right) \cdot q^{1}+\left(q^{\lambda}+2+q^{-\lambda}\right) \cdot q^{2}+\ldots\right\}=\frac{1}{\eta(q)} \sum_{n \in \mathbb{Z}} q^{n^{2}+\lambda^{2} / 4} e^{\pi \lambda t n} \\
& =\frac{1}{\eta(q)} \sum_{n \in \mathbb{Z}} q^{(n-\lambda / 2)^{2}} .
\end{aligned}
$$

With the $g$ insertion, we get

$$
\begin{aligned}
\operatorname{tr} g q^{L_{0}-1 / 24} & =q^{\lambda^{2} / 4}\left\{(1) \cdot q^{0}+\left(-q^{\lambda}+1-q^{-\lambda}\right) \cdot q^{1}+\left(-q^{\lambda}+2-q^{-\lambda}\right) \cdot q^{2}+\ldots\right\}=\frac{1}{\eta(q)} \sum_{n \in \mathbb{Z}}(-1)^{n} q^{n^{2}+\lambda^{2} / 4} e^{\pi \lambda t n} \\
& =\frac{1}{\eta(q)} \sum_{n \in \mathbb{Z}}(-1)^{n} q^{(n-\lambda / 2)^{2}} .
\end{aligned}
$$

In the above expressions, we have $q=e^{-\pi t}$. Poisson resummation and modular transformation then gives

$$
\operatorname{tr} q^{L_{0}-1 / 24}=\frac{1}{\eta(q)} \sum_{n \in \mathbb{Z}} e^{-\pi t(n-\lambda / 2)^{2}}=\frac{1}{\sqrt{2} \eta\left(\tilde{q}^{2}\right)} \sum_{m \in \mathbb{Z}} \tilde{q}^{m^{2} / 2} e^{i \pi \lambda m},
$$

where $\tilde{q}=e^{-2 \pi / t}$. Similarly,

$$
\operatorname{tr} g q^{L_{0}-1 / 24}=\frac{1}{\eta(q)} \sum_{n \in \mathbb{Z}}(-1)^{n} q^{(n-\lambda / 2)^{2}}=\frac{1}{\sqrt{2} \eta\left(\tilde{q}^{2}\right)} \sum_{m \in \mathbb{Z}} \tilde{q}^{(m-1 / 2)^{2} / 2} e^{i \pi \lambda(m-1 / 2)} .
$$


[1] C. G. Callan, I. R. Klebanov, A. W. W. Ludwig, and J. M. Maldacena, Nucl. Phys. B422, 417 (1994).

[2] J. Polchinski and L. Thorlacius, Phys. Rev. D 50, R622 (1994).

[3] M. Gutperle and A. Strominger, J. High Energy Phys. 04 (2002) 018.

[4] A. Sen, J. High Energy Phys. 04 (2002) 048.

[5] F. Larsen, A. Naqvi, and S. Terashima, J. High Energy Phys. 02 (2003) 039.

[6] V. Balasubramanian, S. F. Hassan, E. Keski-Vakkuri, and A. Naqvi, Phys. Rev. D 67, 026003 (2003).

[7] R. Biswas, E. Keski-Vakkuri, R. G. Leigh, S. Nowling, and E. Sharpe, J. High Energy Phys. 01 (2004) 064.

[8] S. Kawai, E. Keski-Vakkuri, R. G. Leigh, and S. Nowling, following article, Phys. Rev. D 73, 106004 (2006).

[9] S. Kawai, E. Keski-Vakkuri, R. G. Leigh, and S. Nowling, Phys. Rev. Lett. 96, 031301 (2006).

[10] A. Recknagel and V. Schomerus, Nucl. Phys. B545, 233 (1999).

[11] N.D. Lambert and I. Sachs, J. High Energy Phys. 02 (2001) 018.
[12] M. Oshikawa and I. Affleck, Nucl. Phys. B495, 533 (1997).

[13] J. L. Cardy, Nucl. Phys. B324, 581 (1989).

[14] P. Di Francesco, P. Mathieu, and D. Senechal, Conformal Field Theory (Springer, New York, USA, 1997).

[15] M. R. Gaberdiel and A. Recknagel, J. High Energy Phys. 11 (2001) 016.

[16] N. Lambert, H. Liu, and J. Maldacena, hep-th/0303139.

[17] D. Gaiotto, N. Itzhaki, and L. Rastelli, Nucl. Phys. B688, 70 (2004).

[18] J.L. Karczmarek, H. Liu, J. Maldacena, and A. Strominger, J. High Energy Phys. 11 (2003) 042.

[19] A. Sen, J. High Energy Phys. 05 (2004) 076.

[20] I. Affleck, Acta Phys. Pol. B 26, 1869 (1995).

[21] K. R. Kristjansson and L. Thorlacius, Classical Quantum Gravity 21, S1359 (2004); J. High Energy Phys. 01 (2005) 047.

[22] M. R. Gaberdiel, A. Recknagel, and G. M. T. Watts, Nucl. Phys. B626, 344 (2002); L. S. Tseng, J. High Energy Phys. 04 (2002) 051; M. R. Gaberdiel and M. Gutperle, J. High Energy Phys. 02 (2005) 051. 